\title{
Ménage à trois? - Zur gewandelten Rolle der EZB im Spannungsfeld zwischen Geldpolitik, Finanzaufsicht und Fiskalpolitik
}

\author{
Von Cornelia Manger-Nestler und Robert Böttner, Leipzig*
}

\begin{abstract}
Die Nachwirkungen der jüngsten Finanz- und Staatsschuldenkrise in der Eurozone haben die Notwendigkeit und den Wunsch nach einer verstärkten Finanzaufsicht hervorgebracht, die unter anderem auf einer stärkeren Einbindung der Expertise der Zentralbanken fußt. Auf europäischer Ebene ist hiermit eine immer stärkere Beteiligung der Europäischen Zentralbank (EZB) bei der Bekämpfung und Vermeidung von Krisen verbunden. Die Betrauung der EZB mit neuen Aufgaben im Bereich der Bankenaufsicht und der Finanzpolitik birgt jedoch die Gefahr von Zielkonflikten zwischen diesen neuen Aufgaben und ihrer traditionellen Rolle in der Geldpolitik, allen voran ihrer primären Aufgabe, der Preisstabilität. Der folgende Artikel wirft einen Blick auf die veränderte Rolle der EZB und das sich aus dem Dreieck der Kapazitäten nunmehr entwickelnde Zusammenspiel.
\end{abstract}

\section{Einleitung}

Seit die Finanzmärkte 2008 in Schieflage gerieten, etablierten die Mitgliedstaaten eine Kaskade neuer Finanzaufsichtsstrukturen, die verstärkt auf die Expertise von Zentralbanken zurückgreifen und diese institutionell einbinden. Die Regelungsintensität dieser Maßnahmen reicht von politischen Absichtserklärungen (Fiskalpakt) und verbalen Ankündigungen der EZB (OMT) mit erkennbar „marktberuhigender Wirkung" über mehrere Sekundärrechtspakete zur Finanzaufsicht (ESFS; SSM; SRM) und zur Überwachung makroökonomischer Ungleichgewichte (Six-/Twopack) bis zur Änderung des Primärrechts (Art. 136 Abs. 3 AEUV) als Grundlage für völkerrechtliche Vereinbarungen (ESM-Vertrag). Angesichts der immer aktiveren Rolle, die die EZB beim Krisenmanagement spielt ${ }^{1}$ und die denknotwendig weder ge- noch verboten ist, könnte man faktisch resümieren: „Wer lebt, muß auf Wechsel gefasst sein. “2 Dass damit die Debatte um die juristische wie politökonomische Konformität der gewandelten Position der EZB nicht beendet ist, liegt auf der Hand. Der Beitrag widmet sich daher den Wechselwirkungen zwischen der geldpolitischen Hauptaufgabe und den aufsichtsrechtlichen (unter III.) wie fiskalpolitischen (unter IV.) „Antworten“ der Union. Dazu ist zunächst der primärrechtliche Rahmen zu umreißen (unter II.), der die zielbezogene Aufgabenerfüllung, sprich den Handlungsspielraum der EZB insgesamt, absteckt.

\footnotetext{
* Prof. Dr. iur. Cornelia Manger-Nestler, LL.M., ist Professorin für Deutsches und Internationales Wirtschaftsrecht an der HTWK Leipzig. Robert Böttner, B.A., ist wiss. Mitarbeiter an der Professur.

1 Ausf. O. Morwinsky, Die Rolle der Europäischen Zentralbank in der Staatsschuldenkrise, in: Blanke/Pilz (Hrsg.), Die „Fiskalunion“, 2014, S. $39 \mathrm{ff}$.

2 Goethe, Wilhelm Meisters Wanderjahre, Kap. 1, 3.
} 


\section{Primärrechtliche Zielvorgaben}

\section{Stabilitätsorientierte Geldpolitik}

Geldpolitik umschreibt die Steuerung von Geldangebot und -nachfrage in einem Währungsraum und gehört zu den charakteristischen Aufgaben von nationalen Zentralbanken. ${ }^{3}$ Bei der Konzeption der Währungsunion stand es daher außer Frage, dass die gemeinsame Geldpolitik eines vergemeinschafteten Währungsgebiets (Euroraum) nur durch einheitliche institutionelle Strukturen - etabliert in Gestalt des ESZB (Art. 127 Abs. 2, tir. 1 AEUV) - festgelegt und ausgeführt werden kann. ${ }^{4}$ Das „arbeitsteilige Zusammenwirken“ von EZB bei der Festlegung und nationalen Zentralbanken bei der Durchführung der Geldpolitik ${ }^{5}$ markiert einerseits die Führungsrolle (Art. 129 Abs. 1 AEUV) der EZB innerhalb des ESZB, andererseits die in Mehrebenensystemen typische Aufgabendezentralisierung.

Die Erfüllung der Hauptaufgaben des ESZB steht im Zeichen des quasi-konstitutionellen, weil primärrechtlich bindenden ${ }^{6}$ Stabilitätsziels (Art. 127 Abs. 1 S. 1 AEUV), das uneingeschränkten Vorrang vor anderen wirtschaftspolitischen Zielen genießt (S. 2, 3). Zum Vergleich: Selbst der von der Deutschen Bundesbank verfolgte strikte Stabilitätskurs spiegelte nur eine über Jahrzehnte konsistente Zentralbankpolitik wider, die $\S 3$ BBankG (a.F.) als ,Währungssicherung“ umschrieb. ${ }^{7}$ Aus der strikten Zielpriorität des AEUV folgt hingegen, dass das ESZB im Falle von Gefahren für die Preisstabilität, die als Binnenwährungsstabilität verstanden wird, ${ }^{8}$ zu Gegenmaßnahmen nicht nur berechtigt, sondern sogar verpflichtet ist. ${ }^{9}$

\section{Abgrenzung zu sekundären Zielen}

a) Allgemeines

Neben dem stabilitätspolitischen Mandat nennt der AEUV weitere Ziele, die die EZB verfolgen soll; konkret die allgemeine Wirtschaftspolitik (Art. 127 Abs. 1 S. 2, 3) sowie die Finanzsystemstabilität und Bankenaufsicht (Abs. 5, 6). Die Zielpluralität bedeutet nur ein Nebeneinander von Zielen, d.h. daraus resultiert nicht, dass die EZB die sekundären Vorgaben mit derselben Intensität wie das Primärziel verfolgen darf.

3 C. Manger-Nestler, Interaction for Monetary and Financial Stability, EYIEL 5 (2014), S. 35 (36); H.J. Hahn/U. Häde, Währungsrecht, 2. Aufl. 2010, § 2 Rn. 5.

4 Hahn/Häde (Fn. 3), § 15 Rn. 10; Ch. Waldhoff, in: Siekmann (Hrsg.), EWU-Kommentar, 2013, Art. 127 AEUV, Rn. 32.

5 Art. 12.1., S. 2, 3, 4 i.V.m. Art. 14.3. ESZB-S.

6 B. Kempen, in: Streinz, EUV/AEUV, 2. Aufl. 2012, Art. 127 AEUV, Rn. 4; M. Rodi, in: Vedder/Heintschel v. Heinegg (Hrsg.), Europäisches Unionsrecht, 1. Aufl. 2012, Art. 127 AEUV, Rn. 2.

7 Waldhoff (Fn. 4), Art. 127 AEUV, Rn. 9 f. Weiterführend C. Manger-Nestler, Par(s) inter pares?, 2008, S. 50 ff.

8 U. Häde, in: Calliess/Ruffert (Hrsg.) EUV/AEUV, 4. Aufl. 2011, Art. 127 AEUV, Rn. 3; Waldhoff (Fn. 4), Art. 127 AEUV, Rn. 12; Morwinsky (Fn. 1), S. 39 (46 f.).

9 D. Khan, in: Geiger/Khan/Kotzur, EUV/AEUV, 5. Aufl. 2010, Art. 127 AEUV, Rn. 2. 
Während Zentralbanken, allen voran die Bundesbank, seit den 1970er Jahren ihr Mandat eng interpretierten und klar auf Geldpolitik fokussierten, ${ }^{10}$ erforderten Vielschichtigkeit ebenso wie transnationale Auswirkungen von Risiken jedoch mittlerweile einen Strategie- und Perspektivwechsel beim unionsweiten Krisenmanagement, das seither über konventionelle nationalstaatliche Reaktionen hinausreicht. Dies schlägt sich gerade auch in einem gewandelten Selbstverständnis der EZB bei der Interpretation von Zielvorgaben nieder: Neben der geldpolitischen Funktion übernahm die EZB Verantwortung bei der Aufsicht über Systemrisiken im gleichnamigen europäischen Ausschuss (ESRB) ${ }^{11}$ und wird zukünftig zur zentralen Aufsichtsbehörde im einheitlichen Aufsichtsmechanismus (SSM). ${ }^{12}$ Schließlich agiert die EZB fiskalpolitisch, in dem sie Liquidität für angeschlagene Bankensysteme in der Peripherie Europas zur Verfügung stellt und damit konditionierte Fiskalpolitik betreibt, die im Rahmen eines unbegrenzten Anleiheankaufprogramms (Outright Monetary Transactions, OMT) zumindest de facto die Möglichkeit eines lender of last resort eröffnet. ${ }^{13}$

\section{b) Verhältnis zur Wirtschafts- und Fiskalpolitik}

Die EZB darf ausdrücklich die allgemeine Wirtschaftspolitik in der Union unterstützen (Art. 127 Abs. 1 S. 2 AEUV); dies schließt konjunktursteuernde fiskalpolitische Elemente ein, ${ }^{14}$ verbietet aber die einseitige Präferenz nationaler Interessen. ${ }^{15}$ Gerade weil der Geldpolitik aus gesamtwirtschaftlicher Sicht eine dienende Funktion $^{16}$ zukommt (arg. ex Art. 3 EUV), ist eine normierte Zielpräferenz ordnungspolitisch von fundamentaler Bedeutung. Sowohl der Wortlaut von Art. 127 Abs. 1 S. 1 AEUV als auch die Regelungssystematik der S. 2 und 3 statuieren den absoluten Vorrang der Preisstabilität gegenüber anderen Zielen. Im Rahmen des fiskalpolitischen Engagements der EZB ist auf diese Grundaussage zurückzukommen.

\section{c) Aufsicht über Kreditinstitute sowie Finanzsystemstabilität als weitere Aufgaben}

Der in Bezug auf die Finanzaufsicht erweiterte Aufgabenkreis der EZB gründet sich auf die beiden in Art. 127 Abs. 5, 6 AEUV genannten Ziele. Während die mikroprudenzielle Aufsicht akteursbezogen ist, sprich die Tätigkeit einzelner Kreditin-

10 Vgl. zur Diskussion Waldhoff (Fn. 4), Art. 127 AEUV, Rn. 10.

11 S. unten, III.1.

12 S. unten, III.2.

13 Vgl. Morwinsky (Fn. 1), S. 57 f.; s. auch unten, IV.2.

14 Hahn/Häde (Fn. 3), § 15 Rn. 14 ff.

15 S. Griller, in: Grabitz/Hilf/Nettesheim, EUV/AEUV, 2014, Art. 127 AEUV, Rn. 5 f.; Kempen (Fn. 6), Art. 127 AEUV, Rn. 5 f.

16 Häde (Fn. 8), Art. 127 AEUV, Rn. 5. 
stitute im Blick hat, konzentriert sich die systemorientierte makroprudenzielle Aufsicht auf Risiken, die die Finanzstabilität bedrohen. ${ }^{17}$

Trotz der Zielinterdependenzen folgt aus der primärrechtlichen Regelungssystematik nicht, dass beide Vorgaben statisch die Aufgaben derselben Institution, insbesondere der Zentralbank, determinieren. Bereits in den Mitgliedstaaten existieren unterschiedliche Verwaltungsorganisationsmodelle, um anhand europäischer Aufsichtsstandards ${ }^{18}$ und unter mehr oder weniger intensiver Beteiligung der Zentralbank die Tätigkeit der Finanzmarktakteure zu überwachen. Die EZB soll in diesem Zusammenhang nur Unterstützungs- und Koordinationspflichten (Art. 127 Abs. 5 AEUV) sowie explizit von den Mitgliedstaaten übertragene (Abs. 6) Nebenaufgaben ${ }^{19}$ wahrnehmen, die die Befugnisse der primär zuständigen (nationalen wie EU-) Behörden ergänzen.

Ein diesbezüglicher Aufgabenzuwachs, kompetenziell auf Art. 114 (ESRB-VO) bzw. Art. 127 Abs. 6 AEUV (VO 1096/2010) basierend, ${ }^{20}$, wird zunächst an der Rolle der EZB im 2010 errichteten ESRB sichtbar. 2013 übertrug der Unionsgesetzgeber der EZB sodann ausgewählte Bankenaufsichtsaufgaben im neu errichteten SSM. ${ }^{21}$ Obwohl auf Art. 127 Abs. 6 AEUV gestützt, ist fraglich, ob der EZB damit nur ,,besondere“ 22 , d.h. eindeutig beschriebene und genau begrenzte Teilbereiche zugewiesen wurden. Diese restriktive Auslegung des Primärrechts, die den generellen Transfer umfangreicher Aufsichtsaufgaben limitiert, ${ }^{23}$ leitet sich aus den divergierenden Zielen ab: Während die Geldpolitik der Preisstabilität verpflichtet ist, wird die Finanzaufsicht dominiert von der Systemstabilität, die mikro- wie makroprudenziell gesichert werden kann. Werden beide Aufgabenbereiche ein- und derselben Institution übertragen (Zentralbank als Aufsichtsbehörde), ist die Gefahr von Zielkonflikten, auf die noch zurückzukommen sein wird, nur durch eine uneingeschränkte Zielpriorität auszuschließen, die auch bei der Aufgabenerfüllung strikt einzuhalten ist.

17 A.-K. Kaufhold, Der Europäische Ausschuss für Systemrisiken im Finanzsystem als Ausprägung einer neuen Aufsichtsform, Die Verwaltung 46 (2013), S. 21 (26, 51 f.); J. Langner, in: Siekmann (Hrsg.), EWU-Kommentar, 2013, Art. 25 ESZB-S., Rn. 49; C. Manger-Nestler, Impacts of International Law on Restructuring of the Global Financial System, UNYB 15 (2011), S. 165 (193 f.).

18 Vgl. insb. RL 2013/36/EU, ABl. EU 2013 L 176/338, sowie VO (EU) 575/2013, ABl. EU 2013 L 176/1.

19 Waldhoff (Fn. 4), Art. 127 AEUV, Rn. 64, 66.

20 VO (EU) 1092/2010, ABl. EU 2010 L 331/1 (ESRB-VO); VO (EU) 1096/2010, AB1. EU 2010 L 331/162 (Übertragung besonderer Aufgaben bezüglich der Arbeitsweise des ESRB).

21 VO (EU) 1024/2013, ABl. EU 2013 L 287/63 (SSM-VO).

22 Vgl. zur Auslegung des Begriffs: Grabitz/Hilf/Nettesheim/Griller (Fn. 15), Art. 127 AEUV, Rn. 60; Calliess/ Ruffert/Häde (Fn. 8), Art. 127 AEUV, Rn. 56; Streinz/Kempen (Fn. 6), Art. 127 AEUV, Rn. 25; Manger-Nestler (Fn. 7), S. 266; Siekmann/Waldhoff(Fn. 4), Art. 127 AEUV, Rn. 72. Dagegen R. Smits, in: Groeben/Schwar$z e$ (Hrsg.), EUV, EGV, 6. Aufl. 2003, Art. 105 EGV, Rn. 74.

23 Ausf. M. Lehmann/C. Manger-Nestler, Einheitlicher Europäischer Aufsichtsmechanismus: Bankenaufsicht durch die EZB, ZBB 2014, S. 2 (6 f.). 


\section{Unabhängigkeit als Strukturprinzip}

Um ihre Aufgaben ordnungsgemäß zu erfüllen, wurde die EZB mit einem hohen Grad an Unabhängigkeit ausgestattet. Die normative Absicherung der Autonomie (insb. Art. 130, Art. 282 Abs. 3 S. 3 AEUV) sichert einerseits deren Zielbezogenheit im Sinne der Rückbindung an die Preisstabilität. ${ }^{24}$ Andererseits charakterisiert sie ein funktionsbezogenes Strukturprinzip (modus operandi), das vor allem für die Geldpolitik als zentrale Pflicht des ESZB gilt. ${ }^{25}$ Dieser Wirkungszusammenhang, der nicht unumstritten ist, ${ }^{26}$ gewährleistet somit aufgabenbezogene Weisungsfreiheit im Sinne einer von Mitspracherechten Dritter unabhängigen Verfügungsgewalt der EZB über den geldpolitischen Instrumenteneinsatz. ${ }^{27}$ Wie vom EuGH bereits 2003 klargestellt, folgt aus der funktionellen Autonomie, dass Unionseinrichtungen die sich aus der Aufgabenerfüllung der EZB ergebenden Besonderheiten angemessen berücksichtigen müssen ${ }^{28}$.

Da Art. 130 AEUV jegliche äußere Einflussnahme auf die Geldpolitik verhindern soll, erfasst die Norm nicht diejenigen Zuständigkeiten, die der EZB zusätzlich übertragen werden können. ${ }^{29}$ Daraus kann indes nicht geschlossen werden, dass die Übertragung von makro-, besonders aber von mikroprudenziellen Aufsichtsbefugnissen auf die EZB mit Art. 130 AEUV ohne Weiteres vereinbar wäre; ${ }^{30}$ auf Einzelheiten dieses Spannungsverhältnisses ist sogleich einzugehen.

\section{Krisenbewältigung durch Systemverbundaufsicht(en)?}

Wie angedeutet, präzisierten die Mitgliedstaaten die Rolle der EZB bei Aufsicht und Überwachung von Finanzmärkten durch Sekundärrecht. Wenngleich sich Aufgaben und Befugnisse der EZB in der Bankenaufsicht (SSM) deutlich von ihrer Funktion bei der Makroaufsicht (ESRB) unterscheiden, handelt es sich beim SSM wie beim ESRB, der Teil des Europäischen Finanzaufsichtssystems (ESFS) ist, um Hybridkonstruktionen ${ }^{31}$, die supranationale Verbundlösungen abbilden. Charakteristisch für diese systemhaften Aufsichtsverbünde, die je nach Aufsichtszweck nur das Eurosystem (SSM; EZB im ESRB) oder die gesamte EU (ESFS/ESRB) reprä-

24 Häde (Fn. 8), Art. 130 AEUV, Rn. 10; Kempen (Fn. 6), Art. 130 AEUV, Rn. 3.

25 Häde (Fn. 8), Art. 130 AEUV, Rn. 8.

26 Vgl. zur Diskussion H. Siekmann, in: Siekmann (Hrsg.), EWU-Kommentar, 2013, Art. 130 AEUV, Rn. 24 ff.

27 E. Gnan/H. Wittelsberger, in: Groeben/Schwarze (Hrsg.), EUV, EG-Vertrag, 6. Aufl. 2003, Art. 108 EGV, Rn. 19; Hahn/Häde (Fn. 3), §20, Rn. 26 ff.

28 EuGH Rs. C-11/00 (Kommission/EZB), Slg. 2003, I-7147, Rn. 135 ff., insb. Rn. 143; vgl. U. Häde, Die Wirtschafts- und Währungsunion im Vertrag von Lissabon, EuR 2009, S. 200 (218).

29 Siekmann (Fn. 26), Art. 130 AEUV, Rn. 69 f.

30 S. dazu Langner (Fn. 17), Art. 25 ESZB-S., Rn. 79 f,; speziell zum SSM M. Herdegen, Europäische Bankenunion: Wege zu einer einheitlichen Bankenaufsicht, WM 2012, S. 1889 (1894 f.); O. Sacarcelik, Europäische Bankenunion: Rechtliche Rahmenbedingungen und Herausforderungen der einheitlichen europäischen Bankenaufsicht, BKR 2013, S. 353 (357); U.H. Schneider, Inconsistencies and unsolved Problems in the European Banking Union, EuZW 2013, S. 452 (456); Ch. Waldhoff/P. Dieterich, Einführung einer gemeinsamen Bankenaufsich auf EU-Ebene, EWS 2013, S. 72 (78).

31 Kaufhold (Fn. 17), S. 21 (22), entwickelte dafür den Begriff Systemaufsicht. 
sentieren, ist jeweils eine starke Position der beteiligten EU-Institutionen, die wiederum durch ratsähnliche Strukturen (SSM; Aufsichtsgremium bzw. EZB-Rat ${ }^{32}$; ESRB: Verwaltungsrat ${ }^{33}$ ) geführt werden, und damit die (28 bzw. 18) Mitgliedstaaten an der Entscheidungsfindung beteiligen. Die Position, die die EZB in diesen Gremien einnimmt (unter 1. und 2.), und die daraus folgenden Wechselwirkungen (unter 3.) sollen nachfolgend genauer betrachtet werden.

\section{Makroaufsicht: Zur Rolle der EZB im ESRB}

$\mathrm{Zu}$ den zentralen Forderungen des de Larosière-Berichts ${ }^{34}$ zählte die Ergänzung einer integrierten mikroprudenziellen Finanzaufsicht um eine Makroperspektive, die Systemrisiken und systemrelevante ${ }^{35}$ Institute stärker kontrolliert. $\mathrm{Zu}$ diesem Zweck wurde der ESRB ${ }^{36}$ im Rahmen des ESFS als eigenes Makroaufsichtsgremium geschaffen. Ausgerichtet auf das finanzaufsichtlich keineswegs neuartige ${ }^{37}$ Ziel ,Finanzstabilität‘ soll er Systemrisiken überwachen und bewerten. ${ }^{38} \mathrm{Um}$ dem Gremium eine möglichst breitgefächerte Palette an aggregierbaren Einflussgrößen zu öffnen, definierte der Unionsgesetzgeber das Systemrisiko sehr weit ${ }^{39}$ und überließ es dem ESRB, anhand seiner Expertise ein eigenes Risikosteuerpult zu etablieren. ${ }^{40}$

Da der ESRB als Koordinationsgremium strukturiert ist, besitzt er keine eigene Rechtspersönlichkeit. ${ }^{41}$ Die Leitung obliegt dem Verwaltungsrat, der die unionale (EZB, ESAs, Kommission) wie die nationale Ebene (Zentralbankpräsidenten) repräsentiert. ${ }^{42}$ In den ersten fünf Jahren seit Gründung steht der EZB-Präsident dem Verwaltungsrat des ESRB vor. ${ }^{43}$ Alle Mitglieder des Verwaltungsrats sind zur Unparteilichkeit verpflichtet, ${ }^{44}$ weshalb jeder Versuch der Einflussnahme seitens der Unionsorgane oder der Mitgliedstaaten untersagt ist. ${ }^{45}$ Die Unabhängigkeit der Mitglieder des ESRB ähnelt daher dem Maßstab der primärrechtlich garantierten Zentralbankautonomie (Art. 130 AEUV). ${ }^{46}$ Die enge organisatorisch-personelle

34 Vgl. The High-Level Group on Financial Supervision, Brüssel, 25.9.2009, S. 1 (39) auf Grundlage der London Declaration der G-20, 4.-5.9.2009, Nr. 3.

35 Vgl. ausf. M. Günther, Systemrelevanz von Finanzinstituten, WM 2010, S. $825 \mathrm{ff}$.

36 Vgl. ausf. Kaufhold (Fn. 17), S. 21 ff.; Langner (Fn. 17), Art. 25 ESZB-S., Rn. 41 ff.; M. Lehmann/C. MangerNestler, Das neue Europäische Finanzaufsichtssystem, ZBB 2011, S. 2 (20 ff.); A. Thiele, Finanzaufsicht, 2014, S. $501 \mathrm{ff}$.

37 Kaufhold (Fn. 17), S. 21 (24 ff.).

38 Art. 3 Abs. 1 sowie Erwgr. 10 ESRB-VO.

39 Vgl. Art. 2 lit. c) sowie präzisierender Erwgr. 9 ESRB-VO. Vgl. Lehmann/Manger-Nestler (Fn. 36), S. 2 (21).

40 Art. 3 II lit. g) ESRB-VO. Vgl. Zu laufend aktualisierten Kriterien und Bewertungen des ESRB http://www.esrb.europa.eu/pub/rd/html/index.en.html (2.12.2014).

41 Erwgr. 15 ESRB-VO.

42 Art. 4 Abs. 2 i.V.m. Art. 6 Abs. 1 ESRB-VO. Vgl. ausf. Kaufhold (Fn. 17), S. 21 (32 ff.).

43 Art. 5 Abs. 1 S. 1 ESRB-VO.

44 Art. 7 Abs. 1, 2 ESRB-VO.

45 Art. 7 Abs. 3 ESRB-VO.

46 Langner (Fn. 17), Art. 25 ESZB-S., Rn. 82 ff.; Manger-Nestler (Fn. 3), S. 35 (60). 
Verbindung zur EZB wird auch beim ESRB-Sekretariat sichtbar, das bei der EZB angesiedelt ist. ${ }^{47}$

Der Blick auf die Aufsichtsbefugnisse zeigt deutlich das differenzierte Verständnis von Mikro- und Makroaufsicht, aus dem der Unionsgesetzgeber unterschiedlich weitreichende (Eingriffs-)Instrumente ableitete. Identifiziert der ESRB systemrelevante Risiken, kann er Risikowarnungen herausgeben sowie Empfehlungen zur Risikominimierung erteilen und diese notfalls veröffentlichen. ${ }^{48}$ Seine nur an hoheitliche Akteure auf nationaler und/oder EU-Ebene ${ }^{49}$ adressierten Warnungen und Empfehlungen sind nicht verbindlich, weshalb der ESRB die Maßnahmen zu deren Umsetzung zwar überwachen (act or explain) ${ }^{50}$, die Nichteinhaltung aber nicht sanktionieren kann. Dies obliegt allein dem Rat, der auch den sog. Krisenfall feststellt. 51

\section{Bankenaufsicht: Zur zukünftigen Rolle der EZB im SSM}

Schon bald nach der Errichtung des ESFS wurde deutlich, dass weitere Integrationsschritte in Richtung einer europäischen Banken(aufsichts)union ${ }^{52}$ folgen müssen. Der als erste Säule errichtete Aufsichtsverbund (SSM) ${ }^{53}$, der zum 4. November 2014 seine Arbeit aufgenommen hat, umfasst neben der EZB die zuständigen nationalen Behörden der Euro-Mitgliedstaaten; gleichzeitig wurde das Verhältnis zwischen EBA und EZB neu geregelt. ${ }^{54}$ Geleitet wird der SSM durch ein Aufsichtsgremium (supervisory board), das als internes Organ der EZB die Planung und Ausführung der übertragenen Aufsichtsaufgaben verantwortet. ${ }^{55}$ Die EZB entsendet vier Vertreter, die keine geldpolitischen Aufgaben erfüllen dürfen, ${ }^{56}$ in das board, das neben dem Vorsitzenden und dem Stellvertreter die (18) nationalen Aufsichtsbehörden abbildet. ${ }^{57}$ Das aufsichtsrechtliche Letztentscheidungsrecht gebührt jedoch dem EZB-Rat, der aufgrund der enumerativen Aufzählung der EZB-Organe (Art. 129 Abs. 1 AEUV) die Beschlussentwürfe des Gremiums billigen muss. ${ }^{58}$

47 Art. 2 und 3 ESRB-VO.

48 Art. 3 Abs. 2 lit. c) (Warnungen) bzw. lit. d) (Empfehlungen) i.V.m. Art. 16 ESRB-VO. Vgl. ausf. Kaufhold (Fn. 17), S. 21 (45 ff.).

49 Art. 16 Abs. 2 S. 1 ESRB-VO.

50 Siehe dazu Art. 17 Abs. 1 ESRB-VO.

51 Art. 3 Abs. 2 lit. e) ESRB-VO. Zur Erklärung des Krisenfalls durch den Rat vgl. Art. 18 Abs. 2 VO (EU) $1093 / 2010$.

52 Vgl. zum missverständlichen Begriff U.H. Schneider, Europäische Bankenunion - ein Etikettenschwindel!, EuZW 2012, S. $721 \mathrm{ff}$.

53 Vgl. J.-H. Binder, Auf dem Weg zu einer europäischen Bankenunion? Erreichtes, Unerreichtes, offene Fragen, ZBB 2013, S. 297 ff.; J.A. Kämmerer, Bahn frei der Bankenunion?, NVwZ 2013, S. 830 ff.; Lehmann/MangerNestler (Fn. 23); Sacarcelik (Fn. 30); G. Schuster, The banking supervisory competences and powers of the ECB, EuZW Beilage 1/2014, S. 3 ff.; Waldhoff/Dieterich (Fn. 30).

54 VO (EU) 1024/2013 (Fn. 21); VO (EU) 1022/2013, AB1. EU 2013 L 287/5 (Änderung der EBA-VO); ausf. $C$. Manger-Nestler, Die Bankenunion, in: Blanke/Pilz (Hrsg.), Die „Fiskalunion“, 2014, S. 299 (322 ff.).

55 Art. 26 SSM-VO.

56 Art. 26 Abs. 5 SSM-VO.

57 Art. 26 Abs. 1 UAbs. 1 S. 1 SSM-VO.

58 Sog. negativer Konsens, Art. 26 Abs. 8 S. 1 SSM-VO. 
Infolge ihres begrenzten Mandats übernimmt die EZB die aufsichtsrechtliche Primärverantwortung, insbesondere die prudenzielle Aufsicht und Überwachung der Kapitaladäquanzanforderungen ${ }^{59}$, für die (128) „,bedeutenden“ ${ }^{60}$, d.h. größten und systemrelevanten Institute nur innerhalb der Eurozone ${ }^{61}$. Für die ,,weniger bedeutenden“, zahlenmäßig aber weit überwiegenden Institute kooperiert sie eng mit der nationalen Aufsichtsebene, ${ }^{62}$ auf deren Expertise sie zweifelsohne angewiesen ist. ${ }^{63}$ Insgesamt verantwortet die EZB zukünftig jede Zulassung und gegebenenfalls den Entzug der Banklizenz eines Kreditinstituts sowie die Beurteilung des Erwerbs qualifizierter Beteiligungen an Instituten im Euroraum. ${ }^{64}$ Dazu verfügt sie über enumerative Eingriffsbefugnisse, die im Einklang mit dem nicht auf die Geldpolitik beschränkten ${ }^{65}$ ECB law (Art. 132 AEUV) stehen. Dieser Instrumentenkasten erlaubt es der EZB, den gesamten aufsichtsrechtlichen „Lebenszyklus“ eines Instituts zu überwachen und zu jedem Zeitpunkt (präventiv ${ }^{66}$; repressiv ${ }^{67}$ ) angemessen und rechtsverbindlich direkt gegenüber dem Institut agieren zu können. Zudem ist die EZB mit Aufsichtsaufgaben betraut, die dem präventiven Krisenmanagement (Sanierungspläne, frühzeitiges Eingreifen) ${ }^{68}$ dienen und die an der - von Art. 127 Abs. 6 AEUV wohl gerade noch gedeckten - Schnittstelle zur Rolle der zukünftigen Abwicklungsbehörde (in Form einer Agentur der Union) im Abwicklungsmechanismus (SRM) ${ }^{69}$ angesiedelt sind.

\section{Schnittmengen und Spannungsfelder}

Der erweiterte Aufgabenkreis der EZB führt zu einer Konstellation, in der sich Geldpolitik, Makroüberwachung sowie Mikroaufsicht triangulär gegenüberstehen. Es ist daher zu untersuchen, inwieweit die Dreiecksbeziehung(en) der genannten Aufgaben zu Synergien, aber auch Spannungen führen können.

59 VO (EU) 575/2013, ABl. EU 2013 L 176/1, sowie RL 2013/36/EU, ABl. EU 2013 L 176/338.

60 Zum Begriff Lehmann/Manger-Nestler (Fn. 23), S. 2 (13 f.).

61 Art. 2 Ziff. 1, Art. 4 Abs. 1 sowie Art. 6 Abs. 4 SSM-VO.

62 S. Art. 14 f. SSM-VO sowie VO (EU) 468/2014 der EZB, ABl. EU 2014 L 141/1.

63 Thiele (Fn. 36), S. 523; vgl. auch Erwgr. 37 SSM-VO.

64 Art. 4 Abs. 1 lit. a) und c) i.V.m. Art. 6 Abs. 4 UAbs. 1 SSM-VO. Zu Einzelheiten vgl. Lehmann/Manger-Nestler (Fn. 23), S. 2 (15).

65 C. Ohler/M Schmidt-Wenzel, in: Siekmann (Hrsg.), EWU-Kommentar, 2013, Art. 132 AEUV, Rn. 11.

66 Vgl. Art. 10 - 13 SSM-VO.

67 Art. $14-18$ SSM-VO.

68 Art. 4 Abs. 1 lit. i) SSM-VO.

69 RL 2014/59/EU zur Festlegung eines Rahmens für die Sanierung und Abwicklung von Kreditinstituten und Wertpapierfirmen, AB1. 2014 L 173/190; VO (EU) 806/2014 zur Festlegung einheitlicher Vorschriften und eines einheitlichen Verfahrens für die Abwicklung von Kreditinstituten und bestimmten Wertpapierfirmen im Rahmen eines einheitlichen Abwicklungsmechanismus und eines einheitlichen Abwicklungsfonds, ABl. 2014 L 225/1; zwischenstaatliches Übereinkommen vom 21. Mai 2014 über die Übertragung von Beiträgen auf den einheitlichen Abwicklungsfonds und über die gemeinsame Nutzung dieser Beiträge Legislative Entschließung des Parlaments v. 15.4.2014, C7-0223/2013 zum VO-Vorschlag (COM 2013/520). RL-Vorschlag (COM 2012/280) vom Rat am 6.5.2014 in 1. Lesung angenommen. Vgl. zum SRM Lehmann/Manger-Nestler (Fn. 23), S. 2 (19 f.); Manger-Nestler (Fn. 54), S. 299 (336 ff.). 


\section{a) Geldpolitik und Makroaufsicht}

Auch wenn die Sicherung der Preisstabilität primär der EZB, die Aufsicht über Systemrisiken hauptsächlich dem ESRB übertragen ist, wirkt die Finanzsystemstabilität als verbindendes Element zwischen beiden. Da die EZB nur eine Unterstützerrolle bei der Systemstabilität besitzt, ${ }^{70}$ genießt der ESRB insofern eine Vorrangstellung ${ }^{71}$. Infolge der institutionell-organisatorischen Verknüpfung von ESRB und EZB (Vorsitz; Sekretariat) entscheiden allerdings häufig dieselben Personen über Währungs- als auch Finanzstabilität. Diese Funktionsdopplungen sind durchaus gerechtfertigt, denn es existieren signifikante Schnittmengen zwischen makroökonomischer Frühwarnung und stabilitätsorientierter Geldpolitik. ${ }^{72}$ Gerade bei der geldpolitischen Analyse, die der Festlegung der Strategie vorgelagert ist, wertet die EZB eine Vielzahl makro- wie mikroprudenzieller statistischer Daten aus. ${ }^{73}$ Es ist daher folgerichtig, diese Daten auch dem ESRB zur Verfügung zu stellen und gleichzeitig den geld- und währungspolitischen Sachverstand ad personam zu nutzen, wenngleich die ESRB-Warnungen nicht auf das Eurosystem (EU-18) beschränkt, sondern für die gesamte Union (EU-28) ausgesprochen werden.

Das Verhältnis zwischen Geldpolitik und Makroaufsicht birgt jedoch auch Konfliktpotential, das die EZB in ein strukturelles Dilemma ${ }^{74}$ bringen kann. Beispielsweise könnte der Zusammenbruch mehrerer systemrelevanter Finanzinstitute die EZB zu geldpolitischen Maßnahmen motivieren, die zwar die Finanzstabilität (wieder)herstellen, möglicherweise aber das Stabilitätsprimat missachten würden. Infolge der engen Einbindung der EZB in das ESRB wird ein derartiger Zielkonflikt gewissermaßen institutionalisiert. Es ist daher an der EZB, den uneingeschränkten Vorrang der Preisstabilität in ihrer aktiven Politik ,zu leben“; im Zweifel unter Vernachlässigung der übrigen Ziele (Art. 127 Abs. 1 S. 2, 3 und Abs. 5, 6 AEUV). Dabei sollte die EZB, ihre Positionen im ESRB auf nachvollziehbare und transparente Kriterien stützen, die - mangels rechtlicher Kontrollmöglichkeiten - den Grundsatz der Information der Öffentlichkeit wahren. ${ }^{75}$ De lege ferenda wäre eine Kompetenzregelung zur Makroaufsicht angezeigt, die die Hauptverantwortung des ESRB klar regelt und von den EZB-Kompetenzen abgrenzt. ${ }^{76}$ Dies würde die intransparente Vermischung von Aufgaben der EZB als Zentralbank und als Sekretariat des ESRB vermeiden helfen.

70 S. oben, III.1.

71 H. Hartig, Die Befugnisse von EZB und ESRB auf dem Gebiet der Finanzsystemstabilität, EuZW 2012, S. 775 (777); Manger-Nestler (Fn. 3), S. 35 (60), betont den „key player“.

72 M. Mayer, Die gegenwärtige und künftige Rolle der Europäischen Zentralbank bei der Verhütung und Bewältigung von Finanzkrisen, ZBB 2011, S. 25 (32), spricht von „notwendigem Minimum“ an Koordination.

73 Art. 5 ESZB-S. S. dazu Hartig (Fn. 71), S. 775 (776).

74 So Kaufhold (Fn. 17), S. 21 (35 f.).

75 So bereits Lehmann/Manger-Nestler (Fn. 36), S. 2 (23).

76 Ebenso Hartig (Fn. 71), S. 775 (778 f.); Manger-Nestler (Fn. 3), S. 35 (60). 


\section{b) Geldpolitik und Bankenaufsicht}

Unabhängige Geldpolitik und Bankenaufsicht innerhalb derselben Institution in Einklang zu bringen, ist kein leichtes Unterfangen. Dabei steht außer Frage, dass eine einheitliche Aufsichtsstruktur in einem gemeinsamen Währungsraum ordnungspolitischen Rationalitäten ${ }^{77}$ folgt. Diese zwischen beiden Bereichen bestehenden Korrelationen ähneln indes einem magnetischen Feld, welchselbiges einerseits aus praktisch identischen Adressaten resultiert, die als refinanzierungsbedürftige und zugleich aufsichtsunterworfene Kreditinstitute von monetären wie prudenziellen Entscheidungen betroffen sind. Aufgrund der unterschiedlichen Zielvorgaben - Preisstabilität (Geldpolitik) und Finanzsystemstabilität (Bankenaufsicht) besteht andererseits zwischen beiden Feldern ein Konkurrenzverhältnis, das sich verstärkt, sofern dieselbe Institution sowohl geldpolitische Refinanzierung als auch Aufsichtsentscheidungen (letzt)verantworten muss. Insofern läuft die Zentralbank Gefahr, die Geldpolitik nicht mit derselben Kompromisslosigkeit zu betreiben, wie wenn sie nicht die primäre Aufsichtsverantwortung tragen würde. Daran ändert auch Art. 25 SSM-VO wenig, der die strikte Trennung von aufsichtsrechtlicher und geldpolitischer Funktion der EZB festlegt, ${ }^{78}$ auch wenn er ein zentrales ordnungspolitisches Postulat normiert. Potenziert wird das Risiko von Zielkonflikten durch den hohen Autonomiegrad ${ }^{79}$, der Gradmesser der stabilitätsorientierten Geldpolitik ist, aber nicht in gleichem Umfang von administrativen Aufsichtsentscheidungen sein kann. Zwar kann die durch die SSM-VO dem EZB-Rat eingeräumte Weisungsfreiheit nicht dem für die Geldpolitik garantierten Autonomiemaßstab entsprechen; dies folgt schon aus der Normenhierarchie zwischen Art. 130 AEUV und Art. 19 Abs. 1, 2 SSM-VO. Allerdings birgt ein hohes Maß an Autonomie den Verlust von Kontrollbefugnissen innerhalb der jeweiligen Exekutive, ${ }^{80}$ was unter demokratischen und rechtsstaatlichen Gesichtspunkten nicht unkritisch ist. Da die Aufsichtsbefugnisse der EZB bei Weitem den Handlungsrahmen der EBA übersteigen, deren Unabhängigkeit durch umfassende parlamentarische Rechenschaftspflichten demokratisch abgefedert wird, stehen die Aufsichtsentscheidungen der EZB unter erheblichem Legitimationsbedarf. ${ }^{81}$ Dies führt zu einem nur schwer (auf)lösbaren Spannungsverhältnis, vor dem die EZB künftig praktisch steht.

Im EZB-Rat kulminieren die Interessenkonflikte. Seine institutionelle Janusköpfigkeit besteht darin, dass identische Personen Aufsichts- als auch davon unbeeinflusste geldpolitische Beschlüsse letztverantworten müssen. Auch wenn beide Bereiche nur in getrennten Sitzungen mit jeweils eigener Tagesordnung behandelt

77 Vgl. dazu Langner (Fn. 17), Art. 25 ESZB-S., Rn. 86; Lehmann/Manger-Nestler (Fn. 23), S. 2 (7 ff.).

78 S. Art. 25 Abs. 1, 2 UAbs. 1 S. 1, 2 SSM-VO.

79 S. zum Problemkreis Herdegen (Fn. 30), S. 1889 (1894 f.); Sacarcelik (Fn. 30), S. 353 (357); Schneider (Fn. 30), EuZW 2013, S. 452 (456); Waldhoff/Dieterich (Fn. 30), S. 72 (78).

80 Vgl. dazu bereits B. Holznagel/P. Schumacher, Funktionelle Unabhängigkeit und demokratische Legitimation europäischer Regulierungsagenturen, FS Säcker, 2012, S. 737 (748 ff.); Ch. Ohler, Finanzmarktregulierung und -aufsicht, in: Ruffert (Hrsg.), Europäisches Sektorales Wirtschaftsrecht, § 10, Rn. 113.

81 Mit Blick auf die Geldpolitik vgl. Morwinsky (Fn. 1), S. 39 (74 ff.). 
werden dürfen, ${ }^{82}$ geraten chinese walls an dieser Stelle an natürliche, weil menschliche Grenzen. ${ }^{83}$ Es ist praktisch kaum vorstellbar, dass die Ratsmitglieder ihre geldpolitische Expertise wie einen Hut an der Garderobe zurücklassen, um mit dem aufsichtsrechtlichen Hut zur nächsten Ratstagung zu erscheinen. Der EZB-Rat muss daher der Versuchung widerstehen, beide Aufgabenbereiche wechselseitig als Rechtfertigungsgrund zu nutzen und dies mit dem jeweiligen Grad an Unabhängigkeit zu begründen. Hält sich die EZB an ihr derzeitiges primärrechtliches Mandat, muss sie der Wahrung der Preisstabilität den uneingeschränkten Vorrang einräumen. ${ }^{84}$ Es ist daher folgerichtig, wenn der EZB-Rat seinen Widerspruch gegen einen Beschlussentwurf des Aufsichtsgremiums, ,insbesondere durch Verweis auf geldpolitische Erwägungen“ begründen darf. ${ }^{85}$ Eine unmittelbar demokratisch legitimierte Instanz wäre indes geeigneter gewesen, Interessenskonflikte von derartig politischer Brisanz zu schlichten.

\section{c) Makro- und Mikroaufsicht}

Bereits aufgrund der unterschiedlichen Aufsichtsperspektive ist das Verhältnis von akteurszentrierter Mikro- und systemorientierter Makroaufsicht nicht spannungsfrei. ${ }^{86}$ Dies gilt besonders, wenn beide Sichtweisen in einer Institution - hier der EZB - zusammengeführt werden. Der Vorteil einer zentralen Aufsicht liegt dabei darin, dass Maßnahmen der Mikro- und Makroaufsicht nahtlos ineinandergreifen können, da Erkenntnisse in Bezug auf Systemrisiken auch für die Einzelaufsicht und deren Rolle bei der Krisenprävention relevant sind. Infolge ihrer statistischen Aufgaben ${ }^{87}$ erhebt und aggregiert die EZB eine Vielzahl von Daten aus der Mikroaufsicht und verfügt daher über einen maßgeblichen Informationsvorsprung bei systemrelevanten Entwicklungen. Dieses Privileg muss die EZB wohldosiert einsetzen, um sich der Gefahr zu großer Abhängigkeiten zu erwehren, gerade weil konkrete Aufsichtsentscheidungen auch Grundlage für die Abwicklung notleidender Institute sein sollen. Zentral ist jedoch die Forderung, dass makro- wie mikroprudenzielle Erwägungen nicht dazu verleiten dürfen, eigene geldpolitische Entscheidungen $\mathrm{zu}$ diskreditieren. Insofern wäre in der $\mathrm{SSM}^{-\mathrm{VO}^{88}}$ eine deutlichere Trennung zwischen der Rolle der EZB im ESRB und ihren Aufsichtsbefugnissen im SSM wünschenswert gewesen. Umgekehrt finden sich in der ESRB-VO keine ausdrücklichen Kollisionsregeln für den Konfliktfall; auch hier sollte bei der anstehenden Überprüfung des Rechtsakts nachgebessert werden.

82 Art. 25 Abs. 4 S. 2 SSM-VO.

83 Kämmerer (Fn. 53), S. 830 (832); Sacarcelik (Fn. 30), S. 353 (355).

84 S. oben, II.1.

85 Art. 26 Abs. 8 S. 6 SSM-VO.

86 Darauf verweist auch Kaufhold (Fn. 17), S. 21 (38).

87 Art. 5 ESZB-S.

88 Art. 5 i.V.m. Art. 25 Abs. 2 SSM-VO. 


\section{Krisenbewältigung durch fiskalpolitische Maßnahmen?}

Die Reform der Systemaufsicht(en) unter Beteiligung der EZB ist indes nur ein Element der Krisenbewältigung. Die Überwachung und Abwicklung von Instituten im Zuge der Bankenunion soll verhindern, dass sich riskante Geldgeschäfte privater Akteure in die Realwirtschaft „,weiterfressen“ und zum Beinahe-Kollaps von Mitgliedstaaten führen. Hauptursache der Translation von der Banken- zur Staatsfinanzkrise war die Volatilität der betroffenen Eurostaaten aufgrund unsolider Haushaltsführung. Dieser zum Großteil in den Konstruktionsfehlern der WWU begründeten Situation soll einerseits durch innere Reformen wie der Stärkung des Stabilitäts- und Wachstumspakts (SWP) begegnet werden ${ }^{89}$; andererseits sollen institutionelle Neuerungen einen präventiven wie reaktiven Mechanismus gegen ausufernde Staatsverschuldung in Kraft setzen.

\section{Institutionelle Fragen}

\section{a) Der Europäische Stabilitätsmechanismus}

Mit dem qua völkerrechtlichen Vertrag vom 2. Februar 2012 errichteten Europäischen Stabilitätsmechanismus (ESMV) ${ }^{90}$ haben die Euro-Staaten eine neue Finanzinstitution geschaffen, die Liquidität für ihre Mitglieder bereitstellen soll, wenn dies zur Wahrung der Finanzstabilität in der Eurozone unabdingbar scheint (Art. 3 ESMV). Nach dem Vorbild des IWF, dessen Verfahren und Expertise (surveillance; conditionality $)^{91}$ dem Mechanismus Pate stand, knüpft der ESM die Gewährung von Finanzhilfen an strenge Auflagen, die sicherstellen sollen, dass die unionsrechtlichen Regelungen u.a. im Hinblick auf solide Haushaltsführung beachtet werden. ${ }^{92}$ Die Finanzhilfen können zur Rekapitalisierung von Finanzinstituten (Art. 15 ESMV) oder allgemein zugunsten des ESM-Mitglieds verwendet werden (Art. 16 ESMV). Neben Sekundärmarktinterventionen (Art. 18 ESMV) ist auch der mit Gewährung eines Darlehens vergleichbare Anleihekauf am Primärmarkt (Art. 17 ESMV) zulässig. ${ }^{93}$

Die Einrichtung des ESM war bereits Gegenstand mehrerer verfassungsgerichtlicher Streitigkeiten. Dabei ging es insbesondere vor dem BVerfG um die Frage, ob durch den ESM-Vertrag ein Regime geschaffen wird, mittels dessen sich der Deut-

89 Dazu ausf. Herm.-J. Blanke, The European Economic and Monetary Union - between vulnerability and reform, Int. J. Public Law and Policy 2011, S. 402 (409 ff.); St. Pilz/H. Dittmann, Perspektiven des Stabilitäts- und Wachstumspakts, ZEuS 2012, S. $53 \mathrm{ff}$.

90 BGBl. II, S. 981.

91 Vgl. dazu Manger-Nestler (Fn. 3), S. 35 (53 ff.).

92 EuGH Rs. C-370/12 (Pringle), Rn. 111, 121, 143; vgl. dazu etwa Herm.-J. Blanke/St. Pilz, Solidarische Finanzhilfen als Lackmustest föderaler Balance in der Europäischen Union, EuR 2014, S. 540 (550 ff.); W. Frenz, ESM-Vertrag europarechtskonform!?, EWS 2013, S. 27 (29 ff.); W. Weiß/M. Haberkamm, Der ESM vor dem EuGH, EuZW 2013, S. 95 (97 ff.).

93 EuGH (Fn. 92), Rn. 140 f.; krit. dazu N. Horn, Die Reform der Europäischen Währungsunion und die Zukunft des Euro, NJW 2011, S. 1398 (1402). 
sche Bundestag seiner Haushaltsverantwortung entäußert und wodurch die ,integrationsfeste Verfassungsidentität des Grundgesetzes“ verletzt würde. Das BVerfG kam zu dem Schluss, dass Deutschland mit dem Beitritt zum ESM keine der Höhe nach unbeschränkte oder nicht hinreichend absehbare Zahlungspflicht eingegangen sei. Etwaige Auslegungen der Regelungen über den revidierten erhöhten Kapitalabruf (Art. 9 Abs. 2 und Abs. 3 S. 1 i.V.m. Art. 25 Abs. 2 ESMV), die eine solche unbegrenzte Zahlungspflicht Deutschlands begründen könnten, sind durch die gemeinsame Erklärung der ESM-Mitglieder vom 27. September 2012 in völkerrechtlich verbindlicher Weise ausgeschlossen. ${ }^{94}$

Auch die unionsrechtliche Vereinbarkeit des ESM-Vertrags, der als internes Abkommen zwischen den EU-Mitgliedstaaten unionsrechtskonform auszulegen ${ }^{95}$ ist, war lange umstritten. Der EuGH entschied dazu, dass der ESM aufgrund seines Zweckes - Wahrung der Finanzstabilität der Eurozone - der Wirtschaftspolitik zuzuordnen sei, deren Koordinierung grundsätzlich den Mitgliedstaaten obliegt (Art. 5 Abs. 1 AEUV).${ }^{96}$ Da der ESM zudem reaktiver Natur ist, kann kein Verstoß gegen die Art. 123, 125 AEUV festgestellt werden, da die Normen präventiv die Gefahr von Staatsverschuldungskrisen verringern sollen. ${ }^{97}$

Auf nationaler Ebene hebt das BVerfG das Verbot der monetären Haushaltsfinanzierung (Art. 123 AEUV) hervor ${ }^{98}$ und betont, dass an den ESM als eine dem öffentlichen Sektor zugehörige Finanzinstitution nach Art. 123 Abs. 1 AEUV keine Kredite durch die EZB vergeben werden dürfen. ${ }^{99}$ Folglich werden die zulässigen Primärmarktinterventionen seitens des ESM durch seine eigene Kapitalausstattung begrenzt; dieser Begrenzung für Anleihekäufe sieht sich die EZB grundsätzlich nicht gegenüber. ${ }^{100}$

Auch im Rahmen des ESM wird der EZB eine nicht unbedeutende Rolle zugedacht. Sie nimmt als Beobachter an den Sitzungen des Gouverneursrats und des Direktoriums teil (Art. 5 Abs. 3 und 6 Abs. 2 ESMV) und bewertet die Dringlichkeit der Stabilitätshilfeersuchen (Art. 4 Abs. 4 ESMV). In Zusammenarbeit mit der Kommission ist sie mit der Aufgabe betraut, die Gefahr für die Finanzstabilität des EuroWährungsgebiets insgesamt oder seiner Mitgliedstaaten sowie den Finanzierungsbedarf des antragstellenden ESM-Mitglieds zu bewerten. Darüber hinaus ist sie als Mitglied der so genannten „Troika“ (neben Kommission und IWF) an der Ausarbeitung von wirtschaftspolitischen Auflagen und deren Überwachung beteiligt (Memorandum of Understanding, Art. 13 ESMV). Damit speist die EZB ihre Expertise

94 BVerfG, 2 BvR 1390/12 et al., Urt. v. 12.9.2012, Rn. 240 f., 253; Urt. v. 18.3.2014, Rn 188 - ESM/Fiskalpakt mit Anm. C. Manger-Nestler/R. Böttner, NJ 2014, S. 202 ff.; vgl. auch Öst. VGH, SV 2-12/18, Urt. v. 16.3.2013, Rn. 83, 102 ff. - ESM; 101 f.; Estn. Oberstes Gericht, 3-4-1-6-12, Urt. v. 12.7.2012 - ESM. Vg1. dazu W. Kahl, Bewältigung der Staatsschuldenkrise unter Kontrolle des Bundesverfassungsgerichts, EuZW 2013, S. 197 (200 ff.).

95 EuGH Rs. C-235/87 (Matteucci), Slg. 1988, I-5589, Rn. 19.

96 Vgl. EuGH (Fn. 92), Rn. 56 f., 60, 64.

97 EuGH (Fn. 92), Rn. 59, 123 ff., 129 ff.

98 BVerfG, 2 BvR 1390/12 et al., Urt. v. 12.9.2012, Rn. 276 - ESM/Fiskalpakt; vgl. auch Rn. $219 f$.

99 Ebd., Rn. 276 ff.; in diesem Sinne sodann EuGH (Fn. 92), Rn. 96.

100 S. unten, IV.2 
in das wirtschaftspolitische Reformprogramm eines Krisenlandes ein. Für den EuGH bewegt sich das noch im Bereich des Zulässigen, da die „der EZB durch den ESM-Vertrag übertragenen Funktionen [...] den verschiedenen Aufgaben [entsprechen], mit denen sie im [AEUV] und in der Satzung des ESZB betraut wird“. 101

\section{b) Der ,Fiskalvertrag“}

Um das Risiko einer erneuten Wirtschafts- und Finanzkrise zukünftig zu reduzieren, sind strengere Bankenaufsichtsregelungen ${ }^{102}$, aber auch die Einhaltung der unionsrechtlich fixierten Haushaltsdisziplin unabwendbar. Letzteres soll auf sekundärrechtlicher Ebene über einen schärferen SWP erreicht werden. Auf völkerrechtlicher Ebene hat sich die Mehrzahl der EU-Mitglieder durch den Vertrag über Stabilität, Koordinierung und Steuerung in der Wirtschafts- und Währungsunion vom 2. März 2012 (kurz „Fiskalvertrag“) ${ }^{103}$ darauf verständigt, die Koordinierung ihrer Wirtschaftspolitiken zu verstärken und die Haushaltsdisziplin zu fördern, um so „die wirtschaftliche Säule der Wirtschafts- und Währungsunion [...] zu stärken“ (Art. 1 VSKS). Kernelement ist die Verpflichtung zur Einführung einer Schuldenbremse auf nationaler Ebene. Die Ratifikation des Fiskalvertrages ist zudem Voraussetzung für die Gewährung von Stabilitätshilfen durch den ESM (5. Erwägung der Präambel).

Da der Fiskalvertrag den Vorrang des EU-Rechts explizit anerkennt (Art. 2 und 3 VSKS), bestehen diesbezüglich keine Bedenken. Auch von nationaler Ebene wird dem Fiskalpakt abschließend grünes Licht gegeben. Der Regelungsgehalt des Vertrages, insbesondere seines Titels III („Fiskalpolitischer Pakt"), entspricht in weiten Teilen den deutschen verfassungsrechtlichen Vorgaben (sog. „Schuldenbrem$\mathrm{se}^{\text {" } 104}$ ) sowie den europarechtlich existierenden Verpflichtungen, namentlich Art. 126 AEUV und Protokoll Nr. 12. Der Vertrag führt somit auch nicht zu einer Erweiterung der Kompetenzen der EU-Organe. ${ }^{105}$ Ein unmittelbarer Durchgriff der Unionsorgane auf die nationale Haushaltsgesetzgebung ist im Vertrag nicht vorgesehen, insbesondere wird die Budgetverantwortung nicht auf die Europäische Kommission übertragen, sondern verbleibt bei den nationalen Parlamenten. ${ }^{106}$

\section{Aktuelle Entwicklungen im fiskalpolitischen Handlungsspielraum der EZB}

Durch die jüngsten Reformen ist die EZB neben der originären Aufgabe der Geldpolitik nunmehr auch mit Aufgaben der Finanzaufsicht sowie der Fiskal- und Wirt-

101 EuGH (Fn. 92), Rn. 165

102 S. oben, III.

103 BGBl. II, S. 1006. Dazu ausf. St. Pilz, Europa auf dem Weg zur Stabilitätsunion?, DÖV 2012, S. 909 ff.

104 Vgl. dazu U. Kramer/T. Hinrichsen/Th. Lauterbach, Die Schuldenbremse des Grundgesetzes, JuS 2012, S. $896 \mathrm{ff}$.

105 BVerfG (Fn. 98), Rn. 300 ff. - ESM/Fiskalpakt.

106 BVerfG (Fn. 98), Rn. 311 - ESM/Fiskalpakt.; vgl. auch Conseil Constitutionnel, Entscheidung Nr. 2012-653 DC vom 9.8.2012, Erw. Nr. 15. 
schaftspolitik im Rahmen des ESM betraut. Ein solches kompetenzielles Triptychon mag zwar für die EZB als Teil des ESZB zulässig sein (Art. 119 Abs. 2, Art. 127 Abs. 1 S. 2, Abs. 6 AEUV) ${ }^{107}$, aber auch nur, soweit dies ,ohne Beeinträchtigung des Zieles der Preisstabilität möglich ist". ${ }^{108}$ Gerade im Spannungsfeld mit ihrem neuen Verständnis in der Fiskalpolitik läuft die EZB jedoch Gefahr, ihr geldpolitisches Mandat zu vernachlässigen.

Insofern nimmt es nicht Wunder, dass das von der EZB angekündigte OMT-Programm ${ }^{109}$ zum Gegenstand heftiger (auch verfassungsrechtlicher) Auseinandersetzungen wurde. Mit diesem Instrument will die EZB Staatsanleihen von Krisenstaaten notfalls in unbegrenztem Umfang (vom Sekundärmarkt) ankaufen. Durch solch eine umfassende fiskalpolitische Maßnahme drohen indes die Umrisse des bei der EZB angesiedelten Stabilitätsprimats zu zerfasern. Den Gegenentwurf dazu liefert das U.S. Federal Reserve (System) als klassisches Beispiel für ein Vorgabenbündel, das neben monetären Zielen, wie stabilen Preisen und langfristig moderaten Zinsen, auch wirtschaftspolitische (z.B. hohes Beschäftigungsniveau) nennt ${ }^{110}$. Ein derartig breitgefächertes Mandat ist demnach auch der Hintergrund der angelsächsischen unconventional monetary policy, die die Möglichkeit einer direkten Nachfragesteuerung sowie geldpolitische Orientierungen im Sinne von forward guidance ${ }^{111}$ einschließt. Die OMT-Ankündigung könnte ein Hinweis darauf sein, dass die EZB auch bezüglich forward guidance mittlerweile einen Kurswechsel ${ }^{112}$ anstrebt, denn sie nutzt die ,zukunftsgerichteten Hinweise“ als Kommunikationsinstrument, um eine transparente Orientierungshilfe über die künftige geldpolitische Ausrichtung zu geben und somit die Erwartungen der Marktteilnehmer zu stabilisieren. ${ }^{113}$

Inwieweit insbesondere der OMT-Beschluss vom Mandat der EZB (noch) umfasst ist, beschäftigt seit einiger Zeit deutsche wie europäische Gerichte. ${ }^{114}$ Im Januar 2014 entschied das BVerfG, dem EuGH mehrere Fragen zur Vereinbarkeit von OMT mit dem Unionsrecht zur Vorabentscheidung vorzulegen. Das BVerfG legt in seinem Vorlagebeschluss klar dar, dass der OMT-Beschluss nicht mehr vom Mandat der EZB gedeckt sei; es handele sich nicht mehr um eine währungspolitische, sondern überwiegend wirtschaftspolitische Maßnahme, die über die bloße Unterstützung der Wirtschaftspolitik (Art. 127 Abs. 1 S. 2 AEUV) hinausgehe. ${ }^{115}$

107 Zur Wahl der Rechtsgrundlage vgl. Lehmann/Manger-Nestler (Fn. 23), S. 2 (5 ff.).

108 S. oben, II.1.

109 Beschluss des EZB-Rates v. 6.9.2012 zu Technical features of Outright Monetary Transactions, basierend auf der Ankündigung des EZB-Präsidenten v. 2.8.2012.

110 Section 2A, Federal Reserve Act. Vgl. auch Manger-Nestler (Fn. 3), S. 33 (35 f.)

111 Vgl. aus ökonomischer Sicht grundlegend B. Woodward, Interest and Prices, Foundations of a Theory of Monetary Policy, Princeton, 2003, chapter 1.

112 Vgl. allgemein zur Rolle der EZB in der Finanzkrise Mayer (Fn. 72), S. 25 (27 ff.).

113 Deutsche Bundesbank, Monatsbericht August 2013, S. 30 (31). Allg. zur Rolle der EZB in der Krise Mayer (Fn. 72), S. 25 (27 ff.).

114 EuG, Rs. T-492/12 - v. Storch et al., Rn. 32, 51 f., das die Klage mangels Klagebefugnis zurückwies. S. dazu Anm. Steinbach, EuZW 2014, S. 159.

115 BVerfG, 2 BvR 2728/13 et al., Beschluss v. 14.1.2014, Rn. 69 ff., 80 ff. - OMT. Kritisch dazu F. Mayer, Rebels without a cause? - Zur OMT-Vorlage des Bundesverfassungsgerichts, EuR 2014, S. 473 (476 ff.); A. Thiele, Die EZB als fiskal- und währungspolitischer Akteur, EuZW 2014, S. 694 ff. 
Zudem könnte das OMT-Programm gegen das Verbot der monetären Haushaltsfinanzierung (Art. 123 AEUV) verstoßen. ${ }^{116}$ Dass OMT dazu beitragen kann, die währungspolitische Zielsetzung des ESZB zu unterstützen und zudem mit geldpolitischen Mitteln (Offenmarktgeschäfte) umgesetzt werden würde, mache das Programm selbst jedoch nicht schon zu einer zulässigen währungspolitischen Maßnahme. ${ }^{117}$

Das BVerfG vermeidet jedoch die Einstufung als Ultra-Vires-Akt ${ }^{118}$ und schlägt stattdessen eine Art „goldene Brücke“, um den offenen Konflikt mit Luxemburg zu vermeiden. Dies geschieht einerseits durch den Vorlagebeschluss selbst und die verbindliche Anerkennung der Auslegung durch den EuGH, andererseits durch eine subtile Einschränkung seitens des BVerfG seiner spätestens mit dem Lissabon-Urteil begründeten Identitätskontrolle. ${ }^{119}$ Was daraus für den lange beschworenen Rechtsprechungsdialog ${ }^{120}$ zwischen nationaler und unionaler Ebene folgt, bleibt indes abzuwarten. ${ }^{121}$

\section{Fazit}

Die Schaffung neuer Institutionen sowie die stärkere Vernetzung bestehender Strukturen zählt auf nationaler, europäischer wie internationaler Ebene zu den bevorzugten politischen Krisenbewältigungsstrategien. Im Falle der EZB führt deren Einbindung in neue Aufsichtsstrukturen und -mechanismen zu einem grundlegenden Wandel bei Aufgabenumfang und Befugnissen. Dieser Umstand wird dadurch verstärkt, dass die EZB selbst als Teil eines Systems (ESZB) agiert, dessen Mitgliederzahl nicht statisch, sondern dynamisch steigend ist.

Die konstitutionellen Grundlagen der EU-Währungsverfassung fixieren die uneingeschränkte Präferenz der Stabilitätsvorgabe gegenüber anderen, der EZB ebenfalls übertragenen Zielen. Daraus abzuleiten ist zunächst ein verantwortungsvoller Informationsaustausch und Umgang mit Daten, die die EZB aus ihrer geldpolitischen Führungsrolle gewonnen hat und in davon getrennter Funktion in andere Systemaufsichtsverbünde (ESRB; SSM) einbringen soll. Gleichzeitig schaffen die neuen Aufsichtsstrukturen durch systeminterne Pflichten auch Abhängigkeiten, die wiederum die Gefahr der wechselseitigen Beeinflussung von unabhängiger Geldpolitik und (Mikro)Aufsicht bergen. Die EZB ist gefordert, in einem schwierigen Spagat

116 BVerfG (Fn. 115), Rn. 84 ff.

117 BVerfG (Fn. 115), Rn. 96 unter Verweis auf EuGH Rs. C-370/12 (Pringle), Rn. 56. Dagegen Mayer, EuR 2014, S. 473 (477); Thiele, EuZW 2014, S. 694 (695).

118 BVerfG (Fn. 115), Rn. 23, 36 - OMT; vgl. auch Kahl (Fn. 94), S. 197 (199 f.); U. Di Fabio, Karlsruhe Makes a Referral, German Law Journal 2014, S. 107 (109).

119 Vgl. BVerfG (Fn. 115), Rn. 29 und BVerfG, 2 BvE 2/08 et al., Rn. 239 ff. - Lissabon; Herm.-J. Blanke, in: Blanke/Mangiameli (Hrsg.), TEU, 1. Aufl. 2013, Art. 4, Rn. 54 ff.; Kahl (Fn. 94), S. 197 (200 ff.); D. Murswiek, ECB, ECJ, Democracy, and the Federal Constitutional Court, German Law Journal 2014, S. 147 (152 ff.).

120 Dazu etwa Blanke (Fn. 119), Art. 4, Rn. 65; vgl. auch Poln. VGH, SK 45/09, Urt. v. 16.11.2011, sub 2.6; Ital. VGH, 103/2008, Beschluss v. 13.2.2008, Rn. 36; I. Pernice, Der Schutz nationaler Identität in der Europäischen Union, AÖR 2011, S. 185 (218).

121 Vgl. auch Kahl (Fn. 94), S. 197 (205 f.). 
transparente und interessengerechte Entscheidungen zu treffen, die die Zielbezogenheit der jeweiligen Aufgabe respektieren ohne dabei Primärrecht zu verletzen. Dabei birgt ein (zu) weit verstandenes Mandat die Gefahr, dass Maßnahmen zur Krisenbewältigung, die vorübergehend notwendig sein mögen, mit Hilfe monetärer Instrumente bekämpft werden und somit die Unterschiede zwischen der typischerweise unkonditionierten Geldpolitik und konditionierter Fiskalpolitik verwischen. Zudem riskiert die EZB, dass die Bekämpfung nicht monetärer Phänomene mit geldpolitischen Mitteln zu (neuen) fiskalpolitischen Ungleichgewichten führt. Bleibt zu hoffen, dass der EuGH die vom BVerfG konzedierten Grenzen nutzt, um den fiskalpolitischen Aktionsradius der EZB klar zu begrenzen und sie dadurch in diesem Punkt gewissermaßen zu entlasten.

„Gut Ding will Weile haben“122: Zusammenfassend gilt das aus Sicht der EZB für die neue Fülle an Aufsichtsaufgaben, und davon institutionell getrennt, aber zeitlich parallel für die verstärkte Solidarität in der Union, soweit sich alle Beteiligten an die vorher festgelegten Regeln halten. 